\title{
Liver fibrosis-induced muscle atrophy is mediated by elevated levels of circulating TNFa
}

\author{
Tamaki Kurosawa ${ }^{1,2}$, Momo Goto', Noriyuki Kaji ${ }^{3}$, Satoshi Aikiyo', Taiki Mihara', Madoka Ikemoto-Uezumi², \\ Masashi Toyoda (10), Nobuo Kanazawa ${ }^{5}$, Tatsu Nakazawa ${ }^{6}$, Masatoshi Hori ${ }^{1}$ and Akiyoshi Uezumi ${ }^{2}$
}

\begin{abstract}
Liver cirrhosis is a critical health problem associated with several complications, including skeletal muscle atrophy, which adversely affects the clinical outcome of patients independent of their liver functions. However, the precise mechanism underlying liver cirrhosis-induced muscle atrophy has not been elucidated. Here we show that serum factor induced by liver fibrosis leads to skeletal muscle atrophy. Using bile duct ligation (BDL) model of liver injury, we induced liver fibrosis in mice and observed subsequent muscle atrophy and weakness. We developed culture system of human primary myotubes that enables an evaluation of the effects of soluble factors on muscle atrophy and found that serum from BDL mice contains atrophy-inducing factors. This atrophy-inducing effect of BDL mouse serum was mitigated upon inhibition of TNFa signalling but not inhibition of myostatin/activin signalling. The BDL mice exhibited significantly up-regulated serum levels of TNFa when compared with the control mice. Furthermore, the mRNA expression levels of Tnf were markedly up-regulated in the fibrotic liver but not in the skeletal muscles of BDL mice. The gene expression analysis of isolated nuclei revealed that $T n f$ is exclusively expressed in the non-fibrogenic diploid cell population of the fibrotic liver. These findings reveal the mechanism through which circulating TNFa produced in the damaged liver mediates skeletal muscle atrophy. Additionally, this study demonstrated the importance of interorgan communication that underlies the pathogenesis of liver cirrhosis.
\end{abstract}

\section{Introduction}

The liver is involved in various physiological processes, including nutrient metabolism, immune response, detoxification, and digestion. The physiological healing responses to liver injury involve the activation of inflammatory responses and production of extracellular matrix proteins. However, chronic liver injury transforms these physiological healing responses into morbid processes, which lead to the disruption of tissue architecture, loss of hepatic function, and consequently liver cirrhosis $^{1-3}$.

\footnotetext{
Correspondence: Masatoshi Hori (horimasa@g.ecc.u-tokyo.ac.jp) or Akiyoshi Uezumi (uezumi@tmig.or.jp)

${ }^{1}$ Laboratory of Veterinary Pharmacology, Department of Veterinary Medical Sciences, Graduate School of Agriculture and Life Sciences, Tokyo University, 11-1 Yayoi, Bunkyo-ku, Tokyo 113-8657, Japan

${ }^{2}$ Muscle Aging and Regenerative Medicine, Tokyo Metropolitan Institute of Gerontology (TMIG), 35-2 Sakae-cho, Itabashi-ku, Tokyo 173-0015, Japan

Full list of author information is available at the end of the article These authors contributed equally: Tamaki Kurosawa, Momo Goto Edited by M. Agostini
}

Globally, there is increased incidence of mortality due to liver cirrhosis or associated complications among adults $^{4,5}$. The major complications associated with liver fibrosis include hepatocellular carcinoma, variceal bleeding, ascites, and encephalopathy, which contribute to short survival and poor quality of life (QOL) $)^{6}$. In addition to these conditions, muscle atrophy has attracted growing attention recently as a serious complication caused by liver fibrosis ${ }^{7}$. Liver cirrhosis-induced muscle atrophy decreases the QOL of patients and is associated with poor prognosis $^{8-10}$. The Japan Society of Hepatology has established the clinical assessment criteria for muscle atrophy associated with liver disease ${ }^{11}$.

Skeletal muscles mainly comprise large cylindrical cells called myofibers, which are the largest cells in the human body. There are more than 600 skeletal muscles in the human body and each muscle tissue is made up of hundreds of myofibers. Skeletal muscles account for approximately $30 \%$ of the whole-body weight in healthy

\section{(c) The Author(s) 2021}

\footnotetext{
(c) Open Access This article is licensed under a Creative Commons Attribution 4.0 International License, which permits use, sharing, adaptation, distribution and reproduction cc) in any medium or format, as long as you give appropriate credit to the original author(s) and the source, provide a link to the Creative Commons license, and indicate if changes were made. The images or other third party material in this article are included in the article's Creative Commons license, unless indicated otherwise in a credit line to the material. If material is not included in the article's Creative Commons license and your intended use is not permitted by statutory regulation or exceeds the permitted use, you will need to obtain permission directly from the copyright holder. To view a copy of this license, visit http://creativecommons.org/licenses/by/4.0/.
} 
adults. The primary function of skeletal muscle is to mediate body movement through contraction, which enables physical activity and exercise. Additionally, skeletal muscles are a major reservoir of amino acids in the human body and thus play a central role in whole-body protein metabolism ${ }^{12}$. Skeletal muscles are crucial for maintaining the QOL. The progressive decline in skeletal muscle mass and function with ageing results in a condition termed sarcopenia. Sarcopenia not only reduces exercise capacity but also deteriorates general health, and therefore is one of the major determinants of healthy life expectancy. Several studies have indicated that muscle atrophy increases the mortality among the elderly ${ }^{13-16}$. Sarcopenia can be classified as primary and secondary sarcopenia ${ }^{17}$. Ageing is the solo aetiological factor for primary sarcopenia, whereas there are several aetiological factors for secondary sarcopenia in addition to ageing. Liver disease-associated sarcopenia is categorised into secondary sarcopenia and the link between liver disease and sarcopenia has become an increasingly important clinical issue $e^{7,18}$. However, the mechanism that connect these two morbid conditions is not fully understood.

The signalling pathway involved in skeletal muscle atrophy has been well studied. Several extracellular molecules, such as tumour necrosis factor alpha (TNF $\alpha$ ), TNF-like weak inducer of apoptosis (TWEAK), glucocorticoid and myostatin (MSTN) are reported to induce muscle atrophy ${ }^{19,20}$. Patients with liver fibrosis exhibit upregulated expression of MSTN and TNFa ${ }^{21-23}$. MSTN, which is a member of the transforming growth factor beta superfamily, is a negative regulator of muscle mass. Mice with deletion of the Mstn gene exhibit a marked increase in the overall muscle mass ${ }^{24}$, which indicates the negative regulatory effect of MSTN on skeletal muscles. TNF $\alpha$, which is a member of the TNF superfamily, is involved in diverse biological processes, such as inflammation, apoptosis, and tumorigenesis. TNF $\alpha$ exerts its biological function through activation of various intracellular signalling pathways, including JNK, Erk1/2, p38 MAPK, and NF- $\kappa B$ signalling pathways. The activated NF- $\mathrm{kB}$ signalling pathway is reported to induce muscle atrophy through upregulation of MuRF1, a key E3 ubiquitin ligase that mediates sarcomeric protein degradation ${ }^{25}$. Despite these studies, the factors that induce liver fibrosis-induced muscle atrophy have not been completely understood.

In this study, the mechanism underlying liver fibrosisinduced muscle atrophy was elucidated using the bile duct ligation (BDL)-induced liver fibrosis mouse model. The findings of this study indicated that the atrophy-inducing factor is in the serum of BDL mice. The inhibition assays revealed that serum TNF $\alpha$ is an atrophy-inducing factor. Additionally, this study demonstrated that TNF $\alpha$ is produced in the fibrotic liver but not in the atrophied muscle of BDL mice. These findings demonstrate that circulating
TNF $\alpha$ is critical for inter-organ communication, which is important for the pathogenesis of liver cirrhosis-induced muscle atrophy.

\section{Materials and methods \\ Mice}

C57BL/6 and Pdgfra ${ }^{E G F P}$ mice were purchased from Japan SLC (Shizuoka, Japan) and Jackson Laboratory (stock\# 007669, Bar Harbor, ME, USA), respectively. The mice were supplied with food and water ad libitum and maintained under constant temperature and humidity in a 12-h dark/light cycle. All animal experiments were approved by the Experimental Animal Care and Use Committee of University of Tokyo and Tokyo Metropolitan Institute of Gerontology and were performed in accordance with the ARRIVE guidelines.

\section{BDL model}

Eight-week-old male C57BL/6 mice and Pdgfra ${ }^{E G F P}$ mice were randomly divided into BDL and sham operation groups. The BDL group mice were subjected to biliary obstructions as previously described ${ }^{26}$. The common bile duct was exposed through an abdominal incision and ligated at two points under anaesthesia. The sham operation mice underwent the same surgical procedure but the bile duct was not ligated. The abdomen was closed, and the anaesthesia antagonist was used to awaken the mice. The anaesthesia antagonist dose was adjusted according to the method described in a previous study ${ }^{27}$. At day 7 or 21 post-operation, the hindlimb muscles and liver were excised and snap-frozen in liquid nitrogenchilled isopentane.

\section{Etanercept treatment in vivo}

Etanercept (Mochida Pharmaceutical Co., Ltd, Tokyo, Japan) was reconstituted in PBS to a final concentration of $1 \mathrm{mg} / \mathrm{ml}$. Mice were intraperitoneally injected with etanercept $(10 \mathrm{mg} / \mathrm{kg}) 24 \mathrm{~h}$ before surgery, immediately after surgery, and at days 2, 4, and 6 post-operation. Control mice were injected with the same volume of PBS.

\section{Grip strength}

Grip strength of the BDL and sham operation group mouse forelimbs was measured using a grip strength measuring device (GPM-101V, MELQUEST, Toyama, Japan) and (MK380-M, Muromachi Kikai, Tokyo, Japan). The measurement was repeated five times for each mouse and the maximum grip strength was recorded.

\section{Histology}

For immunofluorescence staining, the freshly frozen liver tissues were sectioned into 8 - $\mu \mathrm{m}$-thick sections using cryostat (Leica Microsystems, Wetzlar, Germany). The sections were fixed with $4 \%$ paraformaldehyde (PFA) for 
5 min and blocked with Protein Block Serum-Free (Agilent Technologies, Santa Clara, CA, USA) for $5 \mathrm{~min}$. Next, the sections were incubated with the rabbit anti-collagen type I antibody (1:50; Abcam, Cambridge, UK, Cat\# ab21286) at $4{ }^{\circ} \mathrm{C}$ overnight, followed by incubation with Alexa Fluor 594-conjugated donkey anti-rabbit IgG (1:1000; Jackson ImmunoResearch, West Grove, PA, USA) antibody for $1 \mathrm{~h}$. The sections were counterstained with 4',6-diamidino-2-phenylindole (DAPI) (1:5000; DOJINDO, Kumamoto, Japan) and mounted with SlowFade Diamond antifade reagent (Thermo Fisher Scientific, Waltham, MA, USA). The fluorescent images were captured using a fluorescence microscope DM6000FS (Leica Microsystems). The confocal images were captured using the confocal laser scanning microscopy system TCS SP8 (Leica Microsystems). Collagen type I-positive areas were quantitated using WinROOF2015 (Mitani Corporation, Tokyo, Japan). To analyse the muscle fibre cross-sectional area (CSA), the $8-\mu \mathrm{m}$-thick frozen sections were cut at a position $2.5 \mathrm{~mm}$ from the proximal end of tibialis anterior (TA) muscle. The sections were fixed in ice-cooled acetone for $5 \mathrm{~min}$ and incubated with the rat anti-laminin $\alpha 2$ antibody (clone 4H8-2; 1:200; Santa Cruz Biotechnology, Dallas, TX, USA, Cat\# sc-59854) at $4{ }^{\circ} \mathrm{C}$ overnight, followed by incubation with Alexa Fluor 488-conjugated donkey anti-rat IgG (1:1000; Jackson ImmunoResearch) antibody. Images of entire sections were captured using a fluorescence microscope BZ-X710 (Keyence, Osaka, Japan). CSA and the number of myofibers were quantified using the Hybrid Cell Count software (Keyence). For sequential staining, the cover glasses were removed after immunofluorescence staining, and the same sections were stained with haematoxylin and eosin (H\&E). The images of the sections were captured using a microscope (Optiphot-2, Nikon, Tokyo, Japan) equipped with a CCD camera (DXM1200C, Nikon).

\section{Blood chemistry tests}

The blood samples collected from the BDL or sham groups were incubated at room temperature for $2 \mathrm{~h}$. To collect the serum, the blood samples were centrifuged at $2000 \mathrm{~g}$ for $20 \mathrm{~min}$. The serum level of TNF $\alpha$ was determined using the Mouse TNF $\alpha$ Quantikine HS ELISA kit (R\&D systems, Minneapolis, MN, USA). The serum levels of non-esterified fatty acids (NEFA) were determined by an enzymatic colorimetric method (LabAssay ${ }^{\mathrm{TM}}$ NEFA, Wako, Tokyo, Japan).

\section{Human myotube culture and atrophy assay}

The experiments on primary human cells were approved by the Ethical Committee at Tokyo Metropolitan Geriatric Hospital and Institute of Gerontology. All subjects provided their written informed consent to participate in the study.
Small fragments of muscle tissue were obtained from two human subjects. The human myoblasts were isolated from the gluteus medius or gastrocnemius as previously described $^{28}$. The myoblasts were seeded on Matrigel-coated (CORNING, Corning, NY, USA) 96-well imaging plates (Cell Carrier-96 ultra, PerkinElmer, Waltham, MA, USA) and cultured in growth medium (GM; Dulbecco's modified Eagle's medium (DMEM) supplemented with $20 \%$ foetal bovine serum (FBS), 1\% penicillin-streptomycin, and $2.5 \mathrm{ng} /$ $\mathrm{mL}$ basic fibroblast growth factor (Hygieia Bioscience, Osaka Japan)). The cells were cultured till $90 \%$ confluency at $37^{\circ} \mathrm{C}$ in $5 \% \mathrm{CO}_{2}$ and $3 \% \mathrm{O}_{2}$. The effects of serum from BDL or sham operation group mice and inhibitors on mature myotubes were examined following a previously described method $^{29}$ with minor modifications. The differentiation of myoblasts into myotubes was induced using the differentiation medium (DM; DMEM supplemented with 5\% horse serum and $1 \%$ penicillin-streptomycin). The cells were cultured at $37^{\circ} \mathrm{C}, 5 \% \mathrm{CO}_{2}$, and $20 \% \mathrm{O}_{2}$ for 5 days. The DM was replaced with GM and the cells were cultured for $48 \mathrm{~h}$. The cells were then serum-starved for $4 \mathrm{~h}$ before treatment with sera or inhibitors. The myotubes were incubated with the serum from BDL or sham operation group in the presence or absence of follistatin (R\&D Systems) or TNFRII-Fc protein (R\&D Systems) for 3 days. The myotubes were fixed with $4 \%$ PFA for 5 min and permeabilised with $0.1 \%$ Triton X-100. Next, the myotubes were blocked and incubated with mouse anti-myosin heavy chain (MyHC) antibody (clone MF20; 1:2; DSHB) at $4{ }^{\circ} \mathrm{C}$ overnight, followed by incubation with the Alexa Fluor 647conjugated donkey anti-mouse IgG (1:1000; Jackson ImmunoResearch) antibody for $1 \mathrm{~h}$. The images of the myotubes were captured using the High Content Imaging system IN Cell Analyzer 6000 (Cytiva, Amersham, UK). The MyHC-positive areas were analysed using the IN Cell investigator v.1.9.2 software (Cytiva).

\section{Analysis of the effect of mouse TNFa on human myotubes}

Human myoblasts were induced to differentiate into myotubes in Matrigel-coaled 96-well plates as described above. After serum starvation for $4 \mathrm{~h}$, myotubes were incubated with or without recombinant mouse TNFo (R\&D systems, Minneapolis, MN, USA). The myotubes were fixed with $4 \%$ PFA for $5 \mathrm{~min}$, treated with $0.1 \%$ Triton X-100, blocked and incubated with mouse antimyosin heavy chain (MyHC) antibody (clone MF20; 1,2; DSHB) at $4{ }^{\circ} \mathrm{C}$ overnight. After incubation with the Alexa Fluor 488-conjugated donkey anti-mouse IgG (1:1000; Jackson ImmunoResearch) antibody for $2 \mathrm{~h}$, the images of the myotubes were captured using the High Content Imaging system IN Cell Analyzer 6000 (Cytiva, Amersham, UK). The MyHC-positive areas were analysed using the IN Cell investigator v.1.9.2 software (Cytiva). 


\section{Capillary-based immunoassay}

Cells were lysed in $1 \times$ lysis buffer (RayBiotech, Peachtree Corners, GA, USA) supplemented with cOmplete ULTRA (Roche, Basel, Schweiz) and PhosSTOP (Roche) tablets on ice. The lysates were rotated $15 \mathrm{~min}$ and sonicated for $5 \mathrm{~min}$ at $4{ }^{\circ} \mathrm{C}$. After $15 \mathrm{~min}$ centrifugation at 14,000 r.p.m. at $4{ }^{\circ} \mathrm{C}$, the supernatants were collected as protein lysate samples. The protein concentration of each lysate was determined using Pierce $660 \mathrm{~nm}$ Protein Assay (Thermo Fisher Scientific, Waltham, MA, USA) according to the manufacturer's protocol. The protein concentration was adjusted to 0.5 or $1 \mathrm{mg} / \mathrm{mL}$ using $0.1 \times$ sample buffer 2 (ProteinSimple, San Jose, CA) for the detection of MyHC or MyoD and myogenin, respectively. Protein separation and detection were performed using an automated capillary electrophoresis system Wes (ProteinSimple) with capillary cartridges for $12-230 \mathrm{kDa}$ (ProteinSimple). Following antibodies were used: anti-MyHC (clone MF20; 1:20; DSHB), anti-MyoD (clone EPR6653-131; 1:50; Abcam, Cambridge, UK) and anti-myogenin (clone EPR4789; 1:50; Abcam). Signals were analysed and visualised using Compass software (ProteinSimple).

\section{Nucleus isolation from liver}

Fresh frozen livers of Pdgfra ${ }^{E G F P}$ mice subjected to BDL or sham operation were sectioned into $100-\mu \mathrm{m}$-thick sections using cryostat on day 21 post-operation. The sections $(20 \mathrm{mg})$ were collected into homogenisation tubes (BMS, Tokyo, Japan) containing $3 \mathrm{~mm}$ zirconia beads (BMS). The samples were homogenised in $1 \mathrm{~mL}$ of hypotonic buffer comprising $250 \mathrm{mM}$ sucrose, $10 \mathrm{mM}$ $\mathrm{KCl}, 5 \mathrm{mM} \mathrm{MgCl} 2,10 \mathrm{mM}$ Tris- $\mathrm{HCl}$ (pH 8.0), $25 \mathrm{mM}$ HEPES, $0.1 \mathrm{mM}$ dithiothreitol, 0.1\% Triton X-100, $0.2 \mathrm{U} /$ $\mu \mathrm{L}$ RNase inhibitor (Takara, Shiga, Japan), and protease inhibitor cocktail (Roche, Basel, Schweiz) for $10 \mathrm{~s}$ using a Shakeman homogeniser (BMS). The samples were incubated on ice for $15 \mathrm{~min}$ and homogenised again for $10 \mathrm{~s}$ using the homogeniser. The lysate was filtered through $100-$ and $40-\mu \mathrm{m}$ cell strainers (CORNING), followed by filtration through a $5-\mathrm{mL}$ filter cap FACS tube (CORNING). DAPI (1:5000; DOJINDO) was used to stain the DNA. From each population, 10,000 nuclei were collected using FACSAriaII (BD Biosciences, San Jose, CA, USA). Total RNA was extracted from the samples using the RNeasy Micro kit (Qiagen, Hilden, Germany). The cDNA was synthesised from the extracted RNA using Maxima $\mathrm{H}$ reverse transcriptase (Thermo Fisher Scientific).

\section{RNA extraction and real-time PCR analysis}

Freshly frozen TA muscles and livers were sliced to a thickness of $10 \mu \mathrm{m}$ using a cryostat, and the 100 sections were collected to extract RNA. Total RNA was extracted using the RNeasy Mini kit (Qiagen). The extracted RNA was reverse transcribed into cDNA using the QuantiTect
Reverse Transcription Kit (Qiagen). Real-time PCR analysis was performed using the AriaMx Real-time PCR system (Agilent Technologies) or Takara Thermal Cycler Dice Real-Time System (Takara) with THUNDERBIRD SYBR qPCR Mix (QPS-201, TOYOBO, Osaka, Japan) or SYBR Premix Ex Taq II (Takara). The PCR conditions were as follows: $95^{\circ} \mathrm{C}$ for $60 \mathrm{~s}$, followed by 40 cycles of $95^{\circ} \mathrm{C}$ for $15 \mathrm{~s}, 60^{\circ} \mathrm{C}$ for $20 \mathrm{~s}$, and $72^{\circ} \mathrm{C}$ for $30 \mathrm{~s}$ for tissue samples, or $94^{\circ} \mathrm{C}$ for $30 \mathrm{~s}$, followed by 50 cycles of $94{ }^{\circ} \mathrm{C}$ for $5 \mathrm{~s}, 60^{\circ} \mathrm{C}$ for $20 \mathrm{~s}$, and $72{ }^{\circ} \mathrm{C}$ for $12 \mathrm{~s}$ for isolated nucleus samples. Dissociation curve analysis was performed after amplification. The relative expression level of the target genes was calculated using the $\Delta \Delta \mathrm{Ct}$ method. The Cmas or Gapdh genes were used as reference genes to analyse the target gene expression levels in the tissues or isolated nucleus, respectively. The following primers were used for real-time PCR analysis:

For mouse

Acta2 forward 5'-CGTGCCTATCTATGAGGGCTA

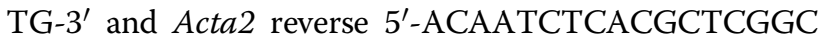
AGTA-3';

Adgre1 forward 5'-GTGGAAGCATCCGAGACACA-3' and Adgre1 reverse 5'-GCTATGGCCAAGGCAAGACA-3';

Ccn 2 forward 5'-CCACCCGAGTTACCAATGAC-3' and $C c n 2$ reverse $5^{\prime}$-ACAGGCTTGGCGATTTTAGG-3';

Cmas forward 5'-CAAAGGCATCCCACTGAAGA- $3^{\prime}$ and Cmas reverse 5'-CCCACACACTCTGGAAGACC-3';

Colla1 forward 5'-CCGAACCCCAAGGAAAAGAA-3' and Colla1 reverse 5'-GTGGACATTAGGCGCAGGAA-3'; Colla2 forward 5'-GCTGGTGTAATGGGTCCTCC-3' and Colla2 reverse 5'-CGACCGGCATCTCCATTAGG-3';

Fbxo32 forward 5'-ACTTCTCGACTGCCATCCTG-3' and $F b x o 32$ reverse $5^{\prime}$-GATGTGAGCTGTGACTTTGCTATC-3';

$F n 1$ forward $5^{\prime}$-CCCAGCTCACTGACCTAAGC- $3^{\prime}$ and Fn1 reverse 5'-TTCTCCTGCCGCAACTACTG-3';

Gapdh forward 5'-CCTGGAGAAACCTGCCAAGTA TG-3' and Gapdh reverse 5'-AGAGTGGGAGTTGCT GTTGAAGTC-3';

Hnf4a forward 5'-AGAGGTTCTGTCCCAGCAGATC-3' and Hnf4a reverse 5'-CGTCTGTGATGTTGGCAATC-3';

$K d r$ forward 5'-GACGTCGACATAGCCTCCAC-3' and $K d r$ reverse $5^{\prime}$-TCGGTGATGTACACGATGCC-3';

Lrat forward 5'-ATGGCTCTCGGATCAGTCCA- $3^{\prime}$ and Lrat reverse 5'-GCCAGGCCTGTGTAGACAAT-3';

Pdgfra forward 5'-TCAGCTGTCTCCTCACAGGG-3' and Pdgfra reverse 5'-ACTCTCCCCAACGCATCTCA-3';

$T g f b 1$ forward $5^{\prime}$-TGAGTGGCTGTCTTTTGACG-3' and $T g f b 1$ reverse $5^{\prime}$-CGTGGAGTTTGTTATCTTTG CTG-3';

Tnf forward 5'-CCACCACGCTCTTCTGTCTAC-3' and $T n f$ reverse 5'-GAGGGTCTGGGCCATAGAACT-3';

Tnfrs 1a: forward 5'-GAAGTTGTGCCTACCTCCTCC$3^{\prime}$ and reverse $5^{\prime}$-GCAAGATAACCAGGGGCAAC- ${ }^{\prime}$ 
Tnfrs 1b: forward 5'-CCAGCCAAACTCCAAGCATC$3^{\prime}$ and reverse $5^{\prime}$-ATGTCACTCCAACAATCAGACC-3' Mstn forward $5^{\prime}$-TGCTGTAACCTTCCCAGGAC- $3^{\prime}$ and $M s t n$ reverse $5^{\prime}$-GATTCCGTGGAGTGCTCATC- ${ }^{\prime}$;

Trim63 forward $5^{\prime}$-TGGAAACGCTATGGAGAACC-3' and Trim63 reverse 5'-AAGATGTCGTTGGCACACT TC-3'.

For human

GAPDH: forward 5'-ACCCACTCCTCCACCTTTGA$3^{\prime}$ and reverse $5^{\prime}$ - TTGCTGTAGCCAAATTCGTTG $-3^{\prime}$

TNFRSF1A: forward $5^{\prime}$-CCCTTCAGAAGTGGGAGG ACAG-3' and reverse 5'-CGAATTCCTTCCAGCGCA ACG-3'

TNFRSF1B: forward $5^{\prime}$-TTGGACTGATTGTGGGTGT G- $3^{\prime}$ and reverse $5^{\prime}$-TTATCGGCAGGCAAGTGAGG-3'

MSTN: forward 5'-GGAGAAGATGGGCTGAATCCG-3' and reverse 5'-CAGCATCGTGATTCTGTTGAGTG-3'.

\section{Statistics}

The effect size for each study was estimated based on a previous study ${ }^{23}$ or preliminary studies. The sample sizes were determined using sample size calculations from the IACUC at Boston university for the type I error rate = 0.05 and power $=0.8$. No data show significant deviation from normal distribution and no data were excluded in this study. Muscle weight measurements were performed in a blinded manner and other experiments were not blinded. All statistical analyses were performed using Prism (version 8.4.1; GraphPad Software, San Diego, CA, USA). Equality of variances between the groups was analysed by $F$-test or Bartlett's test. The differences between two groups were analysed using the two-sided unpaired Student's $t$-test and Welch's correction was applied when variances between the groups was not equal. The differences between more than two groups were analysed using one-way analysis of variance (ANOVA), followed by Dunnett's post hoc test, or Kruskal-Wallis non-parametric test followed by Dunn's post hoc test when variances between the groups was not equal. The differences were considered statistically significant when the $P$ value was less than 0.05 .

\section{Results}

\section{BDL-induced liver fibrosis}

BDL is the most common method used to induce liver fibrosis $^{30,31}$. Eight-week-old male mice were subjected to BDL surgery to induce liver fibrosis. At day 7 postoperation, the connective tissue around the bile ducts thickened and the regular lobular structures in the liver were disrupted (Fig. 1a, lower panels). The accumulation of collagen I around the duct structures in BDL group mice was higher than that in sham operation group mice (Fig. 1a, upper panels and b). The hepatic expression levels of fibrosis-related genes were significantly up-regulated in
BDL group mice (Fig. 1c). At day 21 post-operation, the collagen I-positive fibrotic region was spread throughout the liver in BDL mice (Supplementary Fig. 1). This indicated that BDL efficiently induces liver fibrosis in mice and that the fibrotic changes in the liver are already manifested at least at day 7 post-operation.

\section{$\mathrm{BDL}$ induces skeletal muscle atrophy and weakness}

The phenotype of skeletal muscles during the progression of liver fibrosis induced by BDL was examined. The weight of muscles, including the TA, gastrocnemius (GC), and quadriceps femoris (QF) muscles, decreased in BDL mice at day 3 post-operation. The difference in muscle weight between the BDL and control mice tended to increase after day 3 post-operation (Fig. 2a). The muscle strength was evaluated using the grip test. The change in muscle weight was consistent with the change in grip strength in BDL mice (Fig. 2b). During the process of muscle atrophy, neither the infiltration of inflammatory cells nor myofiber necrosis were observed in both BDL and control mice even at day 21 post-operation (Fig. 2c). Next, the CSA of muscle fibres and number of myofibers were measured. Although each myofiber exhibited atrophy, the number of myofibers was unaffected (Fig. 2d, e). These results indicate that BDL induces liver fibrosis, which promotes muscle atrophy and weakness.

\section{Serum from BDL mice induces myotube atrophy}

As muscle atrophy appeared to be a secondary pathological change following liver fibrosis, we hypothesised that atrophy-inducing factors produced in the fibrotic liver are delivered to the skeletal muscle via the bloodstream. To verify this hypothesis, the cultured myotubes were incubated with the serum collected from BDL or control mice. The serum samples were collected on day 7 post-operation as muscle loss and muscle atrophy were clearly evident at this time point. We first tested $\mathrm{C} 2 \mathrm{C} 12$ mouse myogenic cell line, but myotubes derived from C2C12 cells tended to peel off after treatment with mouse serum for unknown reasons (Supplementary Fig. 2). In contrast, human primary myotubes were stable in culture and therefore suitable for the evaluation of myotube atrophy in vitro. The well-differentiated myotubes derived from highly purified primary human myoblasts were incubated with the serum from BDL or control mice (Fig. 3a). At day 3 post-serum treatment, myosin heavy chain $(\mathrm{MyCH})$ staining revealed that myotubes cultured with BDL mouse serum were significantly thinner than those cultured with control mouse serum (Fig. 3b). Expression levels of MyHC, MyoD1, and myogenin were also examined by capillary-based immunoassay but there was no significant difference in the expression levels of these proteins between sham serumand BDL serum-treated cells (Supplementary Fig. 3), 
a
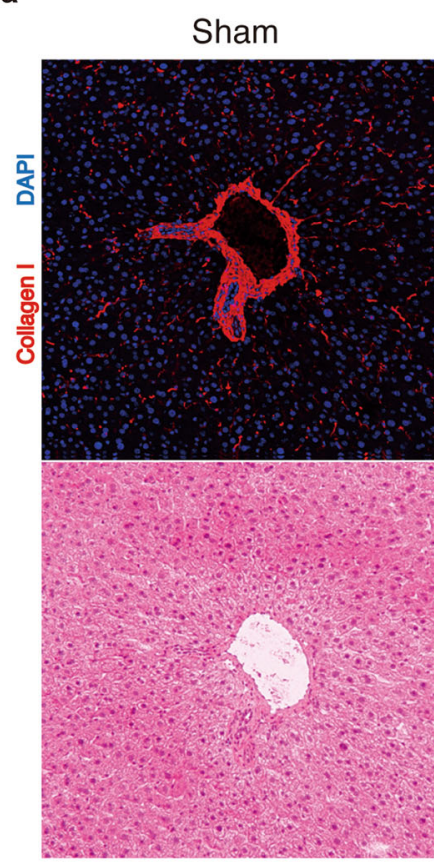

C

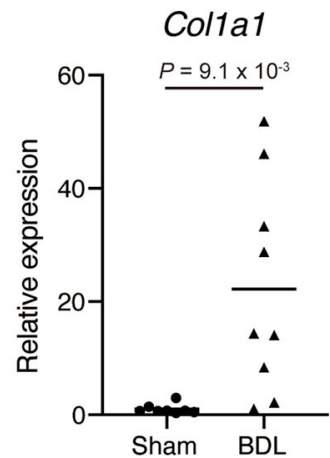

Acta2

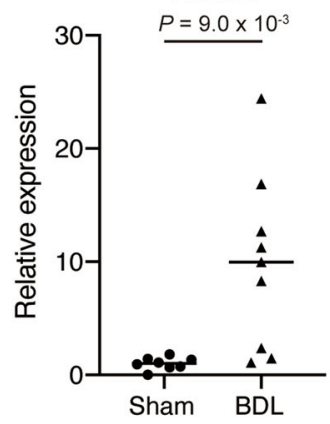

BDL
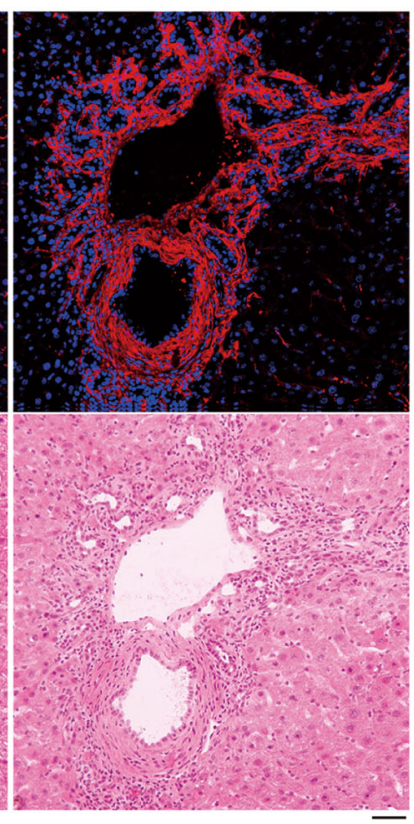

Col1a2

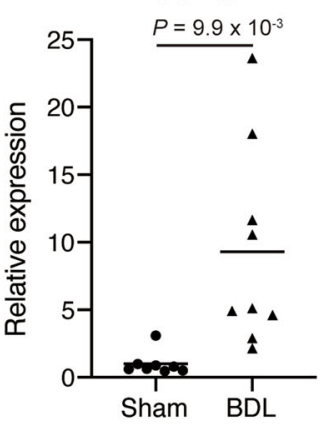

Ccn2

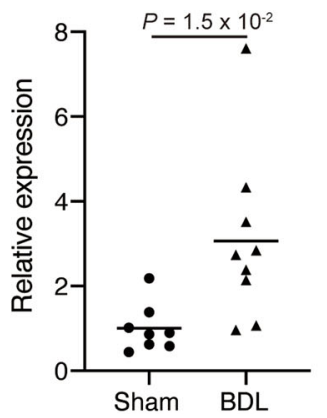

b

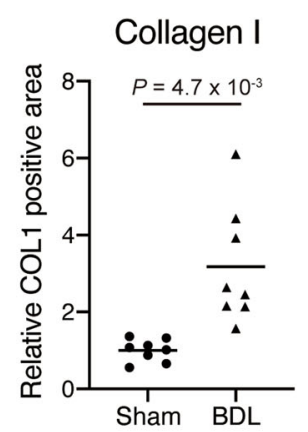

Fig. 1 BDL induces liver fibrosis in mice. The common bile duct of eight-week-old male mice was ligated to induce liver fibrosis. a The sections derived from livers excised on day 7 post-operation were subjected to immunofluorescence staining for collagen I and DAPI (upper) and subsequently to H\&E staining (lower). b The collagen I-positive area was quantified. $n=8$ mice for each group. $\mathbf{c}$ The hepatic expression levels of fibrosis-related genes (Colla1, Col1a2, Fn1, Acta2, Ccn2, and Tgfb1) were quantified on day 7 post-operation. $n=8$ mice for sham and $n=9$ mice for BDL. Data represent individual data points and the means. Data were analysed using the two-sided unpaired t-test and Welch's correction. Scale bar, $50 \mu \mathrm{m}(\mathbf{a})$ 


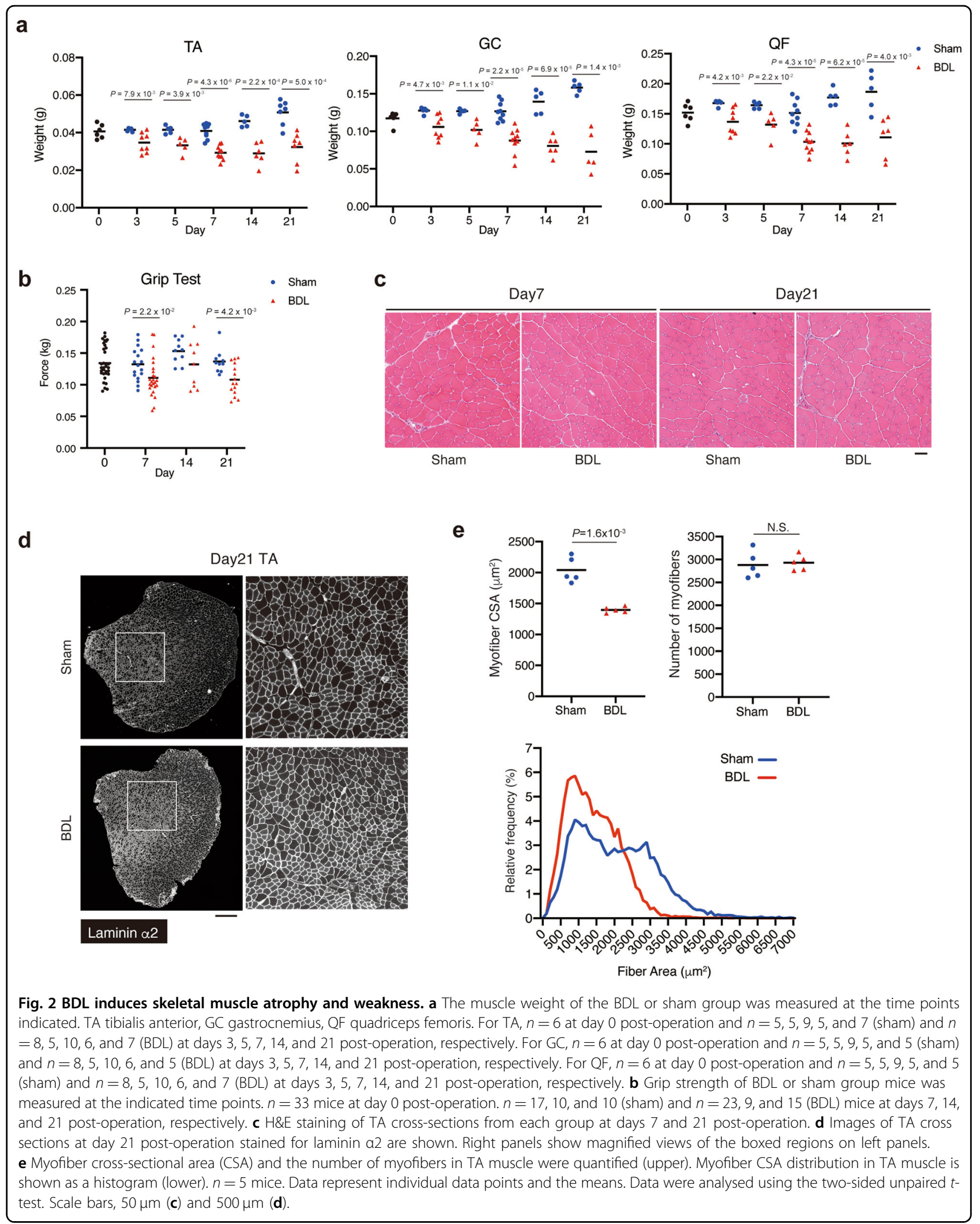




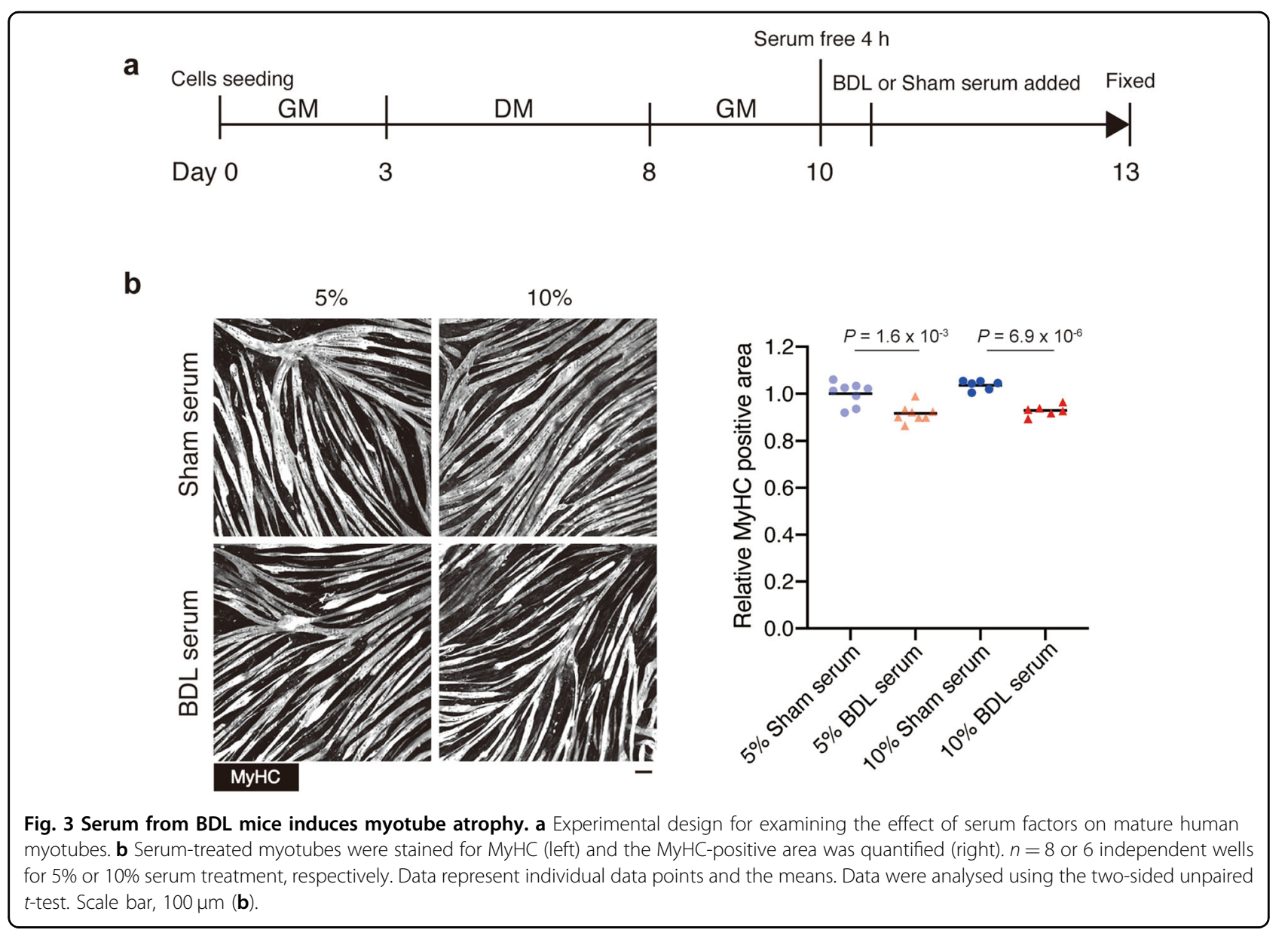

suggesting that morphological analysis based on $\mathrm{MyHC}$ staining is more suitable than protein level analysis to assess myotube atrophy. Muscle atrophy induced by $10 \%$ BDL mouse serum was severer than that induced by $5 \%$ serum (Fig. $3 \mathrm{~b}, P=1.6 \times 10^{-3}$ in $5 \%$ culture vs $P=6.9 \times$ $10^{-6}$ in $10 \%$ culture). These data indicate that the muscle atrophy-inducing factors are present in the serum of mice with liver fibrosis.

\section{TNFa signalling inhibition alleviates myotube atrophy} induced by serum from BDL mice

We sought to identify atrophy-inducing factors that is present in serum derived from the BDL mice. BDL induces loss of adipose tissue (Supplementary Fig. 4a), which may in turn lead to increase in serum levels of free fatty acids. Although fatty acids were reported to induce apoptosis or atrophy in muscle cells ${ }^{32,33}$, it was also reported that lipid overload does not induce lipotoxicity in skeletal muscle ${ }^{34}$. To clarify the involvement of fatty acids in muscle atrophy induced by BDL, serum levels of non-esterified fatty acids (NEFA) were measured. Consistent with previous report ${ }^{35}$, NEFA levels did not differ significantly between control and BDL mice except for day 3 of BDL (Supplementary Fig. 4b). We next focused on other atrophy-related factors, MSTN and TNF $\alpha$, because upregulation of these two factors was observed during liver fibrosis ${ }^{21-23}$. The role of MSTN and TNF $\alpha$ signalling pathways in inducing muscle atrophy was examined using follistatin and TNFRII-Fc chimera protein. Follistatin directly binds to MSTN, activin, and GDF11, which inhibits the binding of these ligands to their receptors. TNFRII-Fc efficiently binds to the TNF $\alpha$ ligand and inhibits the TNF $\alpha$ signalling pathway by functioning as a decoy receptor. Using the same experimental design as used in Fig. 3, the myotube cultures were co-incubated with follistatin or TNFRII-Fc and BDL or control mouse serum (Fig. 4a). Treatment with follistatin did not markedly affect myotube atrophy. BDL mouse serum-induced myotube atrophy was observed even at the highest treatment concentration of follistatin (Fig. 4b). Downregulation rather than upregulation of MSTN expression was observed upon BDL serum treatment, further suggesting that MSTN signalling is not involved in BDL serum-induced myotube atrophy (Fig. 4c). In contrast, TNFRII-Fc suppressed BDL mouse serum-induced myotube atrophy (Fig. 4b). The stronger suppression of 
$\mathbf{a}$

Day 0

b
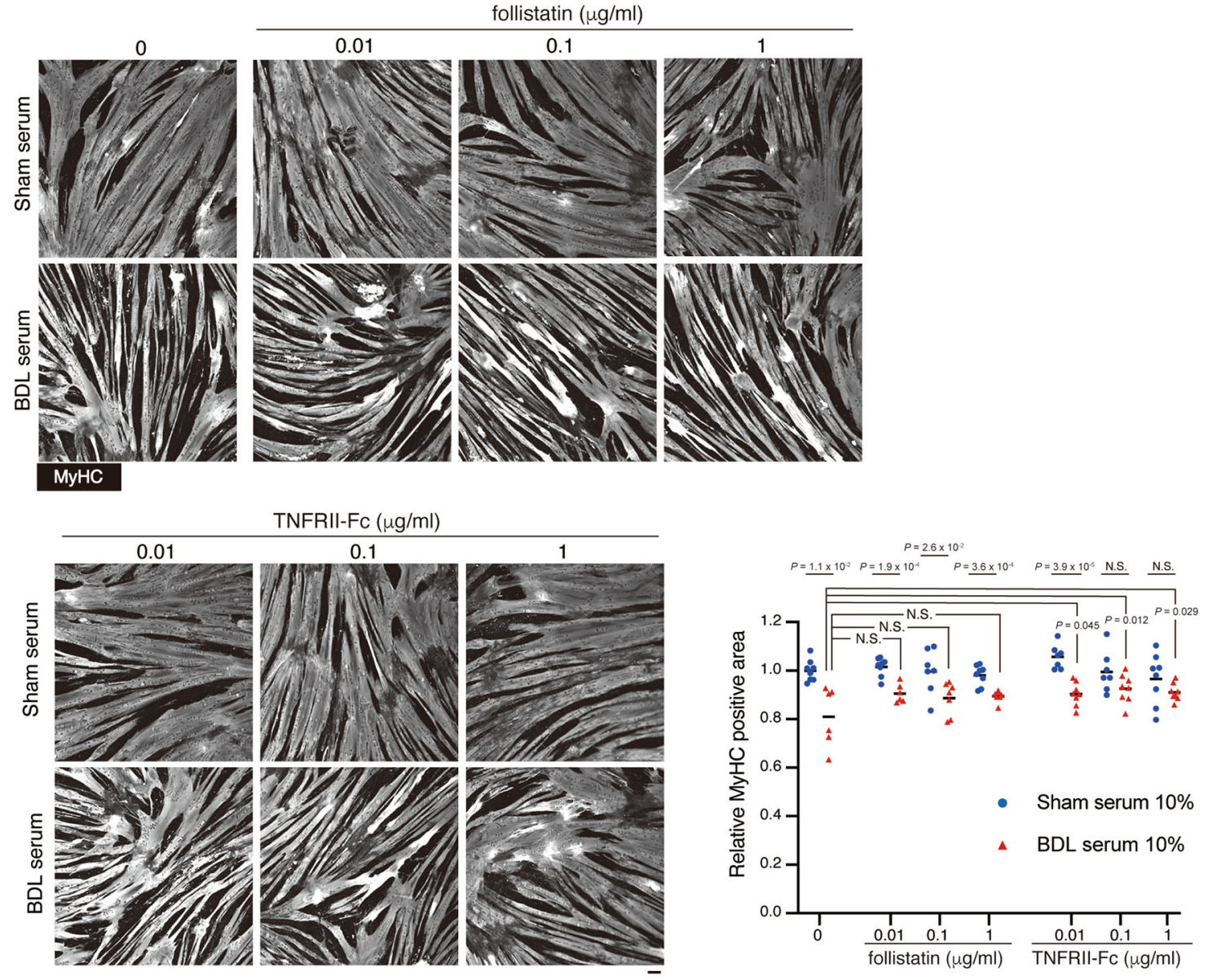

C

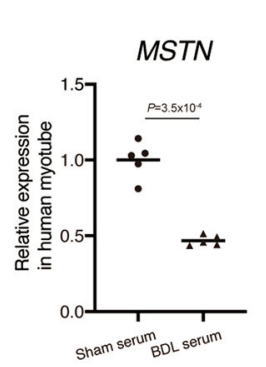

d

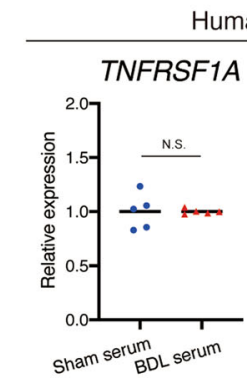

Human myotube

TNFRSF1B

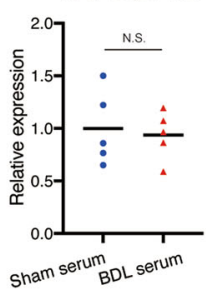

e

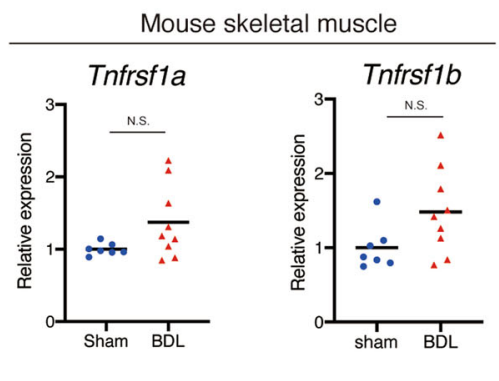

Fig. 4 TNFa is a causative substance of BDL mouse serum-induced myotube atrophy. a Experimental design for examining the effects of MSTN or TNFa inhibition on mature human myotubes treated with BDL serum. $\mathbf{b}$ BDL or sham mouse serum-treated human myotubes were treated with follistatin or TNFRII-Fc at the concentrations indicated. Myotubes were stained for MyHC and the MyHC-positive area was quantified. $\mathbf{c}$ The expression levels of MSTN in the human myotubes treated with sham or BDL serum were quantified. $\mathbf{d}$ The expression levels of TNF receptor genes (TNFRSF1A and TNFRSF1B) in the human myotubes treated with sham or BDL serum were quantified. e The expression levels of TNF receptor genes (Tnfrsf1a and Tnfrsfib) in TA muscles from sham or BDL operation mice were quantified. $n=8$ (sham) and $n=6$ (BDL) independent wells for control (no addition of follistatin or TNFRII-Fc), $n=7,7$, and 8 (sham) and $n=6,7$, and 7 (BDL) for $0.01,0.1$, and $1 \mu \mathrm{g} / \mathrm{mL}$ of follistatin, respectively. $n=7,7$, and 8 (sham) and $n=8,8$, and 8 (BDL) for $0.01,0.1$, and $1 \mu \mathrm{g} / \mathrm{mL}$ of TNFRII-Fc, respectively (b), $n=5$ independent wells (c, d), $n=7$ for sham and 9 for BDL mice (e). Data represent individual data points and the means. Data were analysed using the two-sided unpaired $t$-test, $t$-test with Welch's correction, or ANOVA, followed by Dunnett's post hoc test. NS not significant. Scale bar, $100 \mu \mathrm{m}$ (b). 
atrophy appeared to be achieved with the higher concentration of TNFRII-Fc protein (Fig. 4b). Although there were relatively large variations in $\mathrm{MyHC}$ area of myotubes that had been treated with TNFRII-Fc in addition to sham serum for unknown reasons, myotubes treated with TNFRII-Fc and BDL serum showed a statistically significant increase in $\mathrm{MyHC}$ area compared to myotubes treated with BDL serum alone (Fig. 4b, red triangle). Because human myotubes were used to investigate the effect of BDL mouse serum, we confirmed cross-reactivity of human cells to mouse TNF $\alpha$ by treating human myotubes with recombinant mouse TNF $\alpha$. Addition of mouse TNF $\alpha$ to culture medium clearly induced human myotube atrophy (Supplementary Fig. 5), indicating that human myotubes can react to mouse TNFo. Expression of receptors for TNF $\alpha$ was confirmed in both human myotubes and mouse TA muscle, and expression levels of these receptors did not change after BDL serum treatment or BDL operation (Fig. 4d, e). These results indicate that atrophy-inducing effect of BDL serum can be attributed at least in part to TNF $\alpha$ contained therein.

\section{TNFa expression is up-regulated in the fibrotic liver and not in the skeletal muscle of BDL mice}

The findings so far have suggested that TNF $\alpha$ present in the bloodstream is the causative factor for skeletal muscle atrophy during liver fibrosis. Consistent with these findings, the serum levels of TNF $\alpha$ continued to increase at least until day 7 of BDL (Fig. 5a) with accompanying gradual decrease in muscle weight (Fig. 2a). Inverse relationship between serum TNF $\alpha$ level and muscle mass further supports the importance of TNF $\alpha$ as a causative factor of liver fibrosis-associated muscle wasting. Next, the site of TNF $\alpha$ production was determined. The gene expression level of $T n f$ in the liver was significantly upregulated, whereas that in the skeletal muscle was unaffected in BDL mice (Fig. 5b). Additionally, the cells exhibiting $\operatorname{Tnf}$ expression in the fibrotic liver were examined. A recent study demonstrated that the enzymatic method of cell isolation significantly affects transcriptional and histone modification statuses of isolated cells $^{36}$. Thus, the gene expression was analysed using the isolated nuclei. The fibrotic liver was frozen immediately after harvesting and the nuclei were isolated from the frozen liver to eliminate dissociation-induced transcriptional changes. The isolated nuclei exhibited an intact morphology, which indicated that the nucleus isolation method did not affect the nuclear integrity (Supplementary Fig. 6). Liver-resident macrophages called Kupffer cells were reported to produce TNF $\alpha$ during liver injury $^{37-39}$, while other studies demonstrated that hepatic stellate cells (HSCs), which is the origin of fibrogenic cells in the diseased liver ${ }^{40}$, also produce TNFa ${ }^{41-45}$. To clarify the source of TNF $\alpha$ in our experimental setting, we used the Pdgfra ${ }^{E G F P}$ mice in which the histone H2B-EGFP fusion gene is knocked in at the endogenous Pdgfra locus $^{46}$. These mice enable the identification of nuclei of HSCs, which specifically exhibit Pdgfra expression ${ }^{47}$. Immunofluorescence staining showed that $\mathrm{EGFP}^{+}$cells accumulated in the fibrosis area where collagen I deposition was prominent in the liver at day 21 postoperation (Fig. 5c). The nuclei from the fibrotic liver were fractionated into the following three populations: $\mathrm{EGFP}^{+}$ nuclei, $\mathrm{EGFP}^{-}$diploid nuclei, and $\mathrm{EGFP}^{-}$tetraploid nuclei (Fig. 5d). Specific expression of lecithin-retinol acyltransferase (Lrat), a marker of HSCs, and Pdgfra in $\mathrm{EGFP}^{+}$nuclei ensures accurate labelling of HSCs by the $P d g f r a^{E G F P}$ reporter (Fig. 5e). As expected, the expression levels of fibrogenic genes were highly up-regulated in EGFP $^{+}$nuclei (Fig. 5e). The EGFP ${ }^{-}$fraction comprises diploid and tetraploid nuclei. The expression of genes related to Kupffer cells (Adgre1), endothelial cells (Kdr) and hepatocytes $(H n f 4 a)$ were detected in the EGFP ${ }^{-}$ diploid nuclei. However, the $\mathrm{EGFP}^{-}$tetraploid nuclei exhibited expression of only hepatocyte-related genes (Fig. 5f). Interestingly, the Tnf gene was expressed exclusively in the $\mathrm{EGFP}^{-}$diploid nuclei (Fig. 5g), which indicated that non-fibrogenic diploid cells are the source of TNF $\alpha$ in the fibrotic liver. TNF $\alpha$ is reported to induce muscle atrophy through the upregulation of atrophyrelated E3 ubiquitin ligases, such as atrogin1 and MuRF1 (refs. ${ }^{25,48}$ ). Thus, the expression of Fbxo32 and Trim63 genes that encode atrogin 1 and MuRF1, respectively, was analysed. The expression levels of Fbxo32 and Trim63 were significantly up-regulated in the atrophying muscle of BDL mice at day 7 post-operation (Fig. 5h), implying that the elevated serum level of TNF $\alpha$ leads to muscle atrophy through activation of atrogin1 and MuRF1 pathways. Although follistatin-mediated inhibition of MSTN did not affect myotube atrophy, the expression of Mstn in the skeletal muscles of BDL and control mice was analysed. The Mstn expression levels in the skeletal muscles were not significantly different between BDL and control mice (Fig. 5i). This suggested that MSTN signalling is not involved in muscle atrophy associated with this particular pathological condition.

In vivo blockade of TNFa attenuates loss of muscle mass and function without affecting liver fibrosis in BDL mice

Finally, we performed in vivo blockade of TNF $\alpha$ by etanercept, a clinically approved version of TNFRII-Fc (Fig. 6a). Body weight and serum levels of NEFA were not changed with etanercept treatment (Supplementary Fig. 7). Intriguingly, etanercept treatment attenuated loss of muscle weight and grip strength of BDL-treated mice (Fig. $6 \mathrm{~b}, \mathrm{c})$. Etanercept treatment led to increase in myofiber CSA without affecting myofiber number of BDL-treated mice (Fig. 6d, e), indicating that myofiber atrophy induced 
a

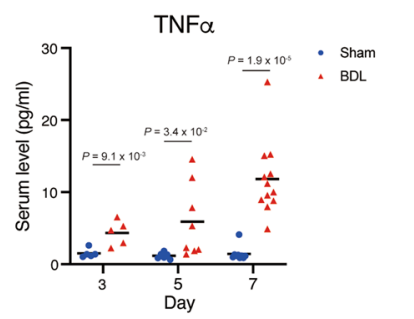

c

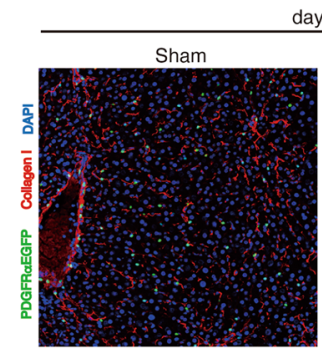

day 21

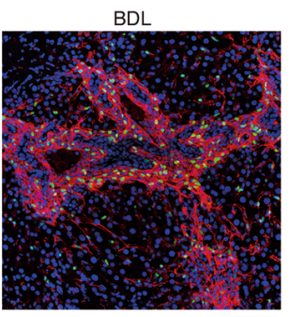

e

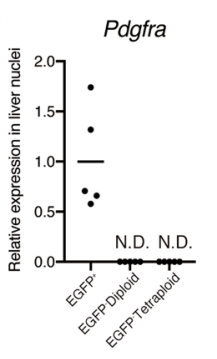

f

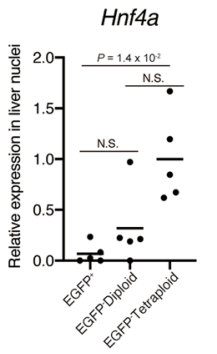

h

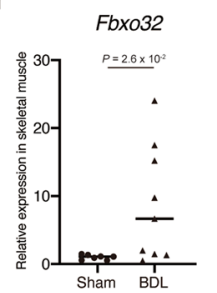

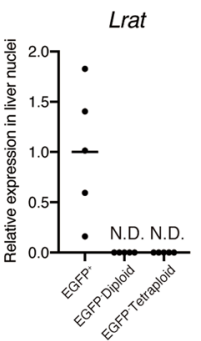
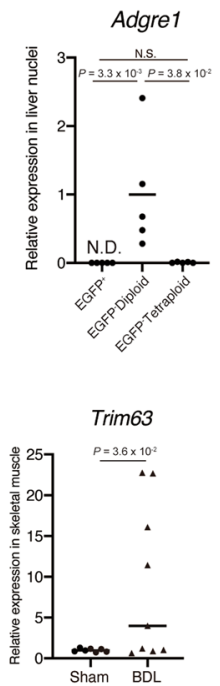

b

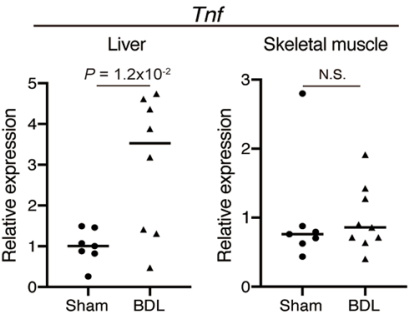

d
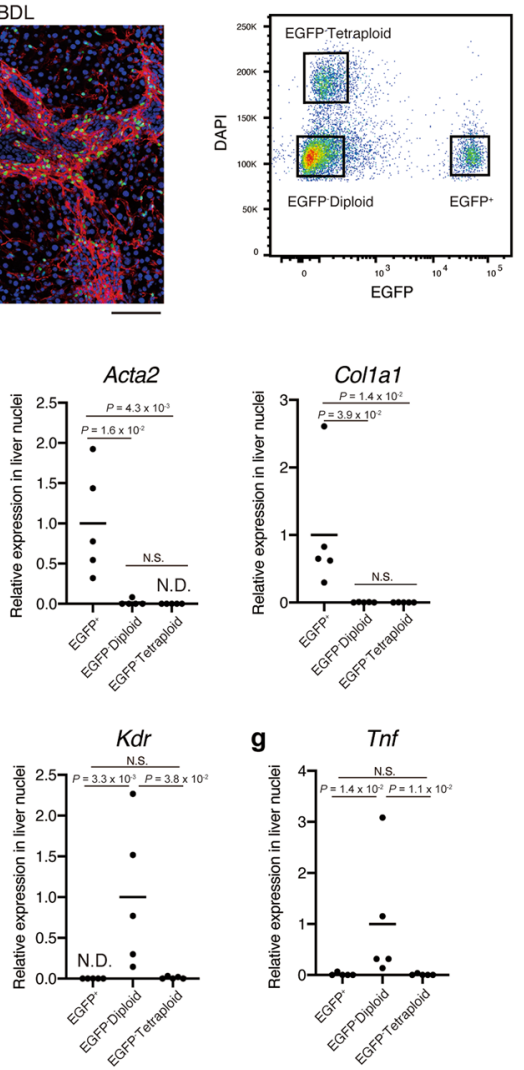

i

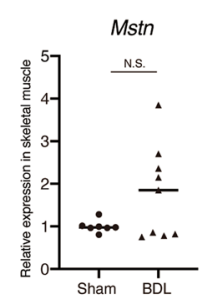

Fig. 5 Tnf expression is up-regulated in the liver but not in the skeletal muscle of BDL mice. a Serum levels of TNFa at day 3, 5, and 7 postoperation were measured using ELISA. $\mathbf{b}$ The expression level of Thf was quantified in the liver (left) and skeletal muscle (right) at day 7 post-operation. c Liver sections of Pdgfra ${ }^{E F P}$ mice at day 21 post-operation were subjected to immunofluorescence staining for collagen I and DAPI. d A representative FACS plot for nuclei isolation. Nuclei were isolated from freshly frozen fibrotic livers of $P$ dgfra ${ }^{E G F P}$ mice using FACS. Three gates were set as indicated in the plot to isolate EGFP+ ${ }^{+}, \mathrm{EGFP}^{-}$diploid, and EGFP- tetraploid nuclei. e The expression levels of hepatic stellate cell markers (Pdgfra and Lrat) and fibrosis markers (Acta2 and Col1a1) were quantified. $\mathbf{f}$ The expression levels of the hepatocyte marker (Hnf4a), Kupffer cell marker (Adgre1), and endothelial cell marker (Kdr) were quantified. $\mathbf{g}$ Quantitative analysis of Tnf gene expression. $\mathbf{h}$ The expression levels of Fbxo32 and Trim63 in the skeletal muscle were quantified. i The expression levels of Mstn in the skeletal muscle were quantified. $n=5,6$, and 9 (sham) and 5, 8, and 12 (BDL) mice for day 3, 5, and 7, respectively (a), $n=7$ (sham) and 8 (BDL) mice for the liver, $n=7$ (sham) and 9 (BDL) mice for the skeletal muscle (b, h, i), and $n=5$ mice $(\mathbf{e}-\mathbf{g})$. Data represent individual data points and the means. Data were analysed using the two-sided unpaired $t$-test, $t$-test with Welch's correction, or Kruskal-Wallis non-parametric test followed by Dunn's post hoc test. NS not significant, ND not detected. Scale bar, $100 \mu \mathrm{m}$ (c). 
a

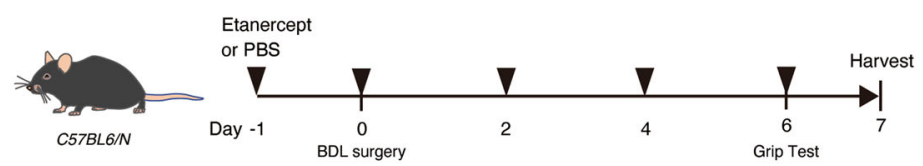

b
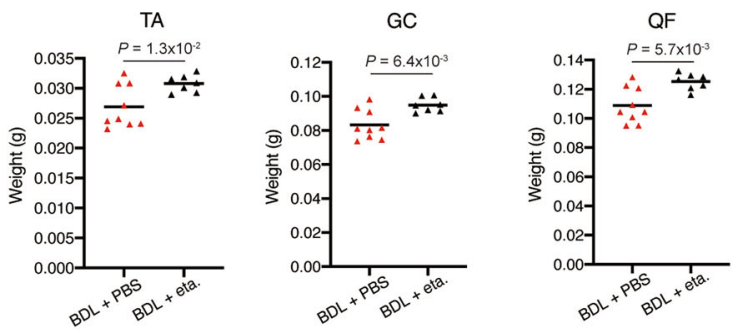

C Grip test

d
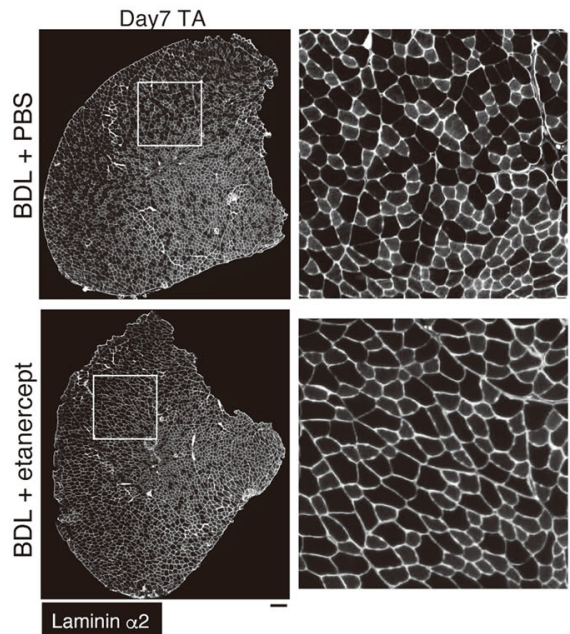

e
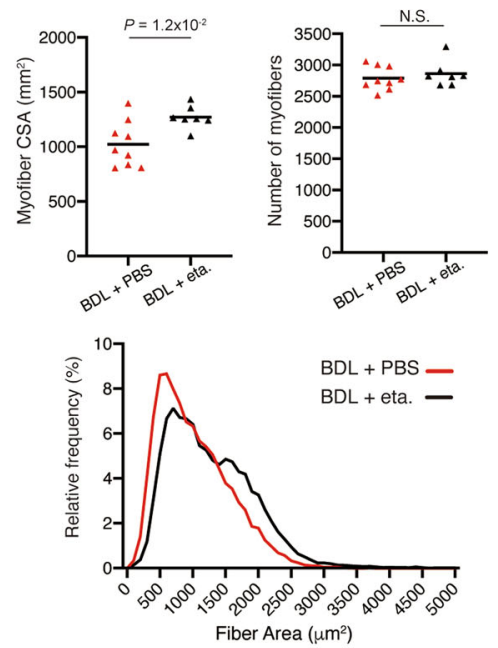

f

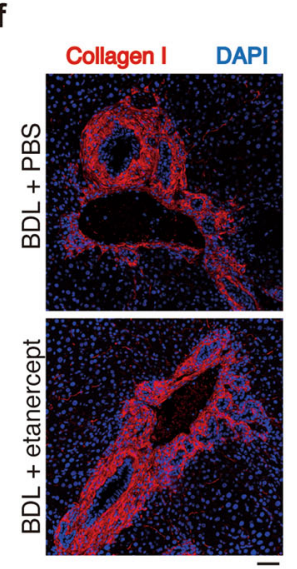

g $\mathbf{h}$

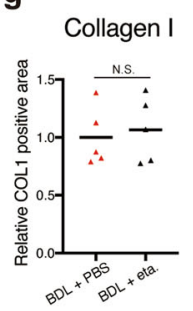

h
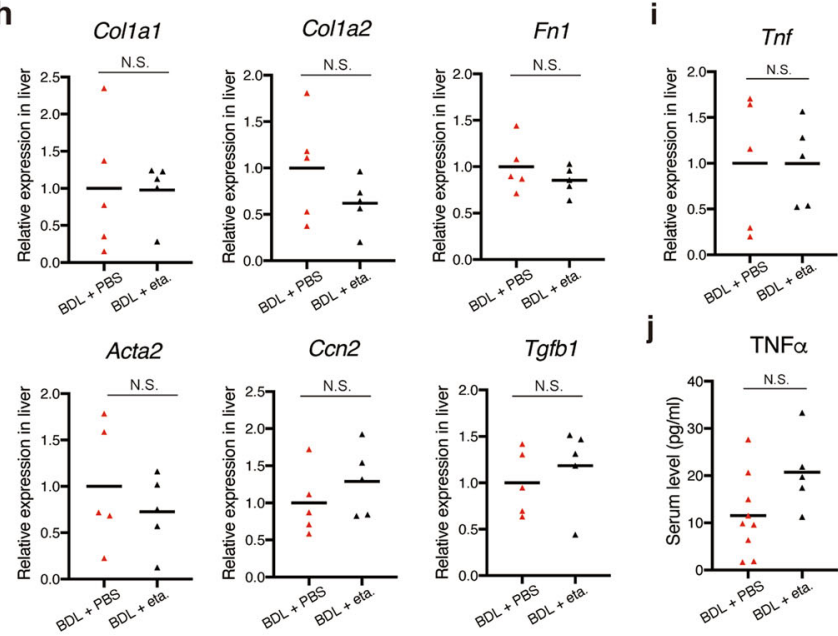

Fig. 6 TNFa inhibition alleviates BDL-induced muscle atrophy. a Experimental design of TNFa blockade by etanercept. $\mathbf{b}$, c The muscle weight (b) and grip strength (c) of the BDL mice treated with PBS or etanercept was measured at day 7. $\mathbf{d}$ TA cross sections of the BDL mice treated with PBS or etanercept were stained for laminin a 2 at day 7 post-operation. Right panels show magnified views of the boxed regions on left panels. e Myofiber CSA and the number of myofibers in TA of BDL mice treated with PBS or etanercept were quantified (upper). Myofiber CSA distribution in TA muscle is shown as a histogram (lower). $\mathbf{f}$ Liver sections of BDL mice treated with PBS or etanercept were subjected to immunofluorescence staining for collagen I and DAPI at day 7 post-operation. $\mathbf{g}$ The collagen I-positive area was quantified. $\mathbf{h}$ The hepatic expression levels of fibrosis-related genes (Colla1, Col1a2, Fn1, Acta2, Ccn2, and Tgfb1) were quantified on day 7 post-operation of BDL mice treated with PBS or etanercept. $\mathbf{i}, \mathbf{j}$ The expression level of Tnf in the liver (i) and the serum levels of TNFa (j) of the BDL mice treated with PBS or etanercept were measured at day 7 post-operation. $n$ $=9$ mice for PBS group and $n=7$ for etanercept group (b, $\mathbf{c}, \mathbf{e})$. $n=5$ mice for each group $(\mathbf{g}, \mathbf{h}, \mathbf{i})$. $n=9$ mice for PBS group and $n=5$ for etanercept group (j). Data were analysed using the two-sided unpaired t-test. Scale bar, $200 \mu \mathrm{m}$ (d) and $50 \mu \mathrm{m}$ (f). 
by BDL is alleviated by TNF $\alpha$ inhibition. Next, effect of etanercept on liver fibrosis was examined. Collagen I deposition and expression levels of fibrogenic genes in the liver were not changed with etanercept treatment (Fig. $6 \mathrm{f}-\mathrm{h})$. Consistent with the result showing non-inhibitory effect of etanercept on liver fibrosis, the gene expression level of $\operatorname{Tnf}$ in the liver and the serum levels of TNF $\alpha$ were not affected by etanercept treatment (Fig. 6i, j). Thus, it appears that etanercept does not exert positive effect on skeletal muscle indirectly through therapeutic effect on liver fibrosis, rather it prevents muscle wasting directly by inhibiting TNF $\alpha$ signalling in skeletal muscle where TNF $\alpha$ is acting as an atrophy-inducing factor. Collectively, our results indicate that TNF $\alpha$ signalling is the mediator of in vivo muscle wasting induced by BDL.

\section{Discussion}

This study revealed the mechanism through which TNF $\alpha$ that is produced in the fibrotic liver and transported to the skeletal muscles through the bloodstream induces muscle atrophy (Fig. 7). This inter-organ crosstalk has clinical significance as there is no treatment for liver disease-induced muscle atrophy. The findings of this study demonstrated that TNFRII-Fc protein-mediated inhibition of TNF $\alpha$ signalling protects the human myotubes or mouse skeletal muscle tissues against atrophy induced by the liver fibrosis. Therefore, inhibiting TNF $\alpha$ signalling in the skeletal muscles where TNF $\alpha$ functions as an atrophy-inducing factor can be an effective preventive strategy for liver fibrosis-induced muscle atrophy. Additionally, TNFRII-Fc, also called etanercept, has been clinically approved and used. Thus, TNFRII-Fc can be a potential therapeutic agent for liver disease-associated muscle atrophy. TNF $\alpha$ signalling inhibition has been evaluated as a potential therapeutic strategy for cancer cachexia in clinical trials. However, the results of these clinical trials are inconsistent with one clinical trial reporting beneficial effects, while other clinical trials reporting no improvement ${ }^{49,50}$. Although not tested in liver disease-associated muscle wasting yet, the strategy that depends on TNF $\alpha$ signal inhibition would have an advantage in liver disease over cancer cachexia. The development of liver fibrosis decreased in mice lacking the type I TNF receptor after repeated $\mathrm{CCl}_{4}$ injury ${ }^{51}$. A recent study on the intrinsic inactive rhomboid protein 2mediated regulation of $\mathrm{TNF} \alpha$ signalling demonstrated that etanercept alleviates BDL-induced liver fibrosis ${ }^{52}$. Although we could not observe inhibitory effect of etanercept on liver fibrosis in our study, discrepancy between studies described above and ours may be attributed to different time point of the analysis. We examined the effect of TNF $\alpha$ inhibition at day 7 of BDL, while former studies analysed the liver 2 or 4 weeks after the induction of liver fibrosis ${ }^{51,52}$. Therefore, the inhibition of TNF $\alpha$

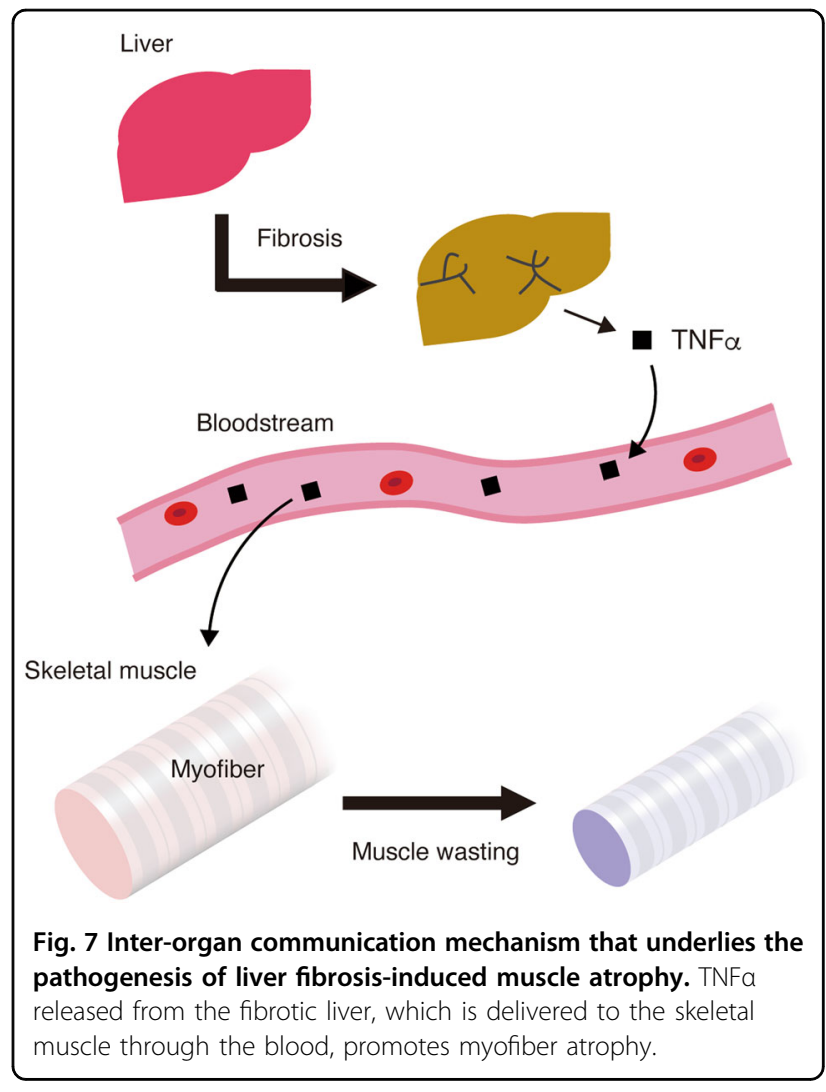

signalling can exert therapeutic effects on liver fibrosis itself at later stage. Thus, TNF $\alpha$ signalling inhibition may alleviate liver fibrosis and the associated complications by exerting an anti-fibrotic effect on the liver and an antiatrophic effect on the skeletal muscle.

The nuclei isolated from the fibrotic liver were subjected to gene expression analysis to determine the source of TNF $\alpha$. Recent studies have revealed that the methods used for cell dissociation affect gene expression. Machado et al. ${ }^{36}$ reported that cell isolation from skeletal muscle through enzymatic digestion markedly affected the transcriptional and histone modification statuses. In the adult kidney, the cell dissociation procedure can damage fragile cells and may not release cells that are embedded within the matrix ${ }^{53}$. Liver is also susceptible to cell dissociationinduced damage. Additionally, it is difficult to recover all cell types with equal integrity and efficiency from the liver tissue $^{54}$. To overcome these limitations, gene expression analysis of isolated intact nuclei is proposed as an alternative approach as nuclei are more resistant to mechanical stress than whole cells and the nuclei can be easily isolated from freshly frozen samples ${ }^{55}$. The analysis of freshly frozen fibrotic liver-derived nuclei revealed that Tnf is exclusively expressed in non-fibrotic diploid cell population, which includes Adgre1-expressing Kupffer cells and $K d r$-expressing endothelial cells, as well as diploid hepatocytes. The Kupffer cells are a potential 
source of TNFa because previous studies have demonstrated the expression of TNFo in Kupffer cells during liver injury ${ }^{37-39}$. Single nucleus RNA sequencing can reveal the TNF $\alpha$-producing cells in fibrotic liver more precisely although this study did not perform this highcost experiment.

Giusto et al. ${ }^{23}$ showed upregulation of MSTN in skeletal muscle of BDL mice but the functional significance of MSTN on muscle atrophy was not examined. Additionally, the authors quantified the protein expression level of MSTN relative to that of actin but actin expression was markedly down-regulated in their study ${ }^{23}$, which indicated that the expression of MSTN was overestimated. In our study, we did not see apparent effect of follistatin on myotube atrophy nor statistically significant upregulation of MSTN in BDL muscle. Although MSTN is a bestknown negative regulator of muscle mass, early clinical trial in muscular dystrophy that directly targets MSTN by neutralising antibody revealed minimal clinical efficacy ${ }^{56}$. Accordingly, novel strategies have been developed to target the MSTN receptor instead of the ligand ${ }^{50}$. Besides MSTN receptor targeting reagents, follistatin is considered as a powerful alternative because it also inhibits activin and GDF11, which are shown to have negative impact on muscle ${ }^{29,57,58}$. In fact, follistatin-mediated inhibition of MSTN/activin signalling pathway further enhances the muscle mass of MSTN-null mice ${ }^{59}$. Nevertheless, treatment with follistatin did not exert a protective effect against myotube atrophy induced by serum from BDL mice in this study, which indicated that the MSTN/activin signalling pathway is not involved in BDLinduced muscle atrophy.

This study demonstrated that TNF $\alpha$ mediates liver fibrosis-induced muscle atrophy. However, the presence of other mechanisms mediating liver fibrosis-induced muscle atrophy cannot be excluded. One of the limitations of our study is the use of human cells to investigate the effect of mouse serum factor. Although we showed that BDL mouse serum induces human myotube atrophy and human myotubes react to mouse TNF $\alpha$, our culture system cannot uncover the effect of factors that are contained in BDL mouse serum but do not cross-react to human cells. In addition, TNFa inhibition did not lead to complete rescue from in vitro myotube atrophy and in vivo muscle atrophy. The TNFRII-Fc protein-treated myotubes still showed bit smaller $\mathrm{MyHC}^{+}$area than the control myotubes and etanercept-treated mice could not exhibit the same level of muscle mass as sham operation mice. Therefore, other cytokines not investigated in this study may be involved in liver fibrosis-induced muscle atrophy. In addition to the effects of cytokines, other mechanisms such as hyperammonaemia may also have an adverse impact on skeletal muscle. Ammonia catabolism through ureagenesis is an important metabolic function of the liver. Hepatocellular damage can trigger ureagenesis impairment and consequently lead to hyperammonaemia. Ammonia accumulated in the muscle is reported to activate molecular events that contribute to sarcopenia ${ }^{60}$. Impaired protein synthesis induced by ammonia is considered to be the molecular mechanism underlying hyperammonaemia-induced muscle atrophy ${ }^{61}$. Elucidating the mediators of liver-muscle axis will improve our understanding of the pathogenesis of liver diseaseinduced muscle atrophy.

In conclusion, this study demonstrated that circulating $\mathrm{TNF} \alpha$, which is released from the fibrotic liver, promotes the atrophy of skeletal muscles during liver fibrosis. Our study shows the molecular mechanism of inter-organ communication that underlies the pathogenesis of liver disease-associated muscle wasting and will provide useful information for designing a therapeutic strategy against this health-threatening complication.

\section{Acknowledgements}

We thank Drs. Kunihiro Tsuchida and Masashi Nakatani for assisting myostatin inhibition experiment, and Heying Zhou for assisting human cell experiment. We thank Editage (www.editage.com) for English language editing. M.H. was funded by Grants-in-Aid for Scientific Research from the Japan Ministry of Education (24248050 and 19H03125). A.U. was funded by JSPS KAKENHI Grant Number JP19H04063, The General Insurance Association of Japan, Research Fund of Mitsukoshi Health and Welfare Foundation, and ONO Medical Research Foundation.

\section{Author details \\ 'Laboratory of Veterinary Pharmacology, Department of Veterinary Medical Sciences, Graduate School of Agriculture and Life Sciences, Tokyo University, 1- 1-1 Yayoi, Bunkyo-ku, Tokyo 113-8657, Japan. ${ }^{2}$ Muscle Aging and Regenerative Medicine, Tokyo Metropolitan Institute of Gerontology (TMIG), 35-2 Sakae-cho, Itabashi-ku, Tokyo 173-0015, Japan. ${ }^{3}$ Laboratory of Veterinary Pharmacology, School of Veterinary Medicine, Azabu University, 1-17-71, Fuchinobe, Chuo-ku, Sagamihara, Kanagawa 252-5201, Japan. ${ }^{4}$ Vascular Medicine, TMIG, Tokyo, Japan. ${ }^{5}$ Department of Surgery, Tokyo Metropolitan Geriatric Hospital and Institute of Gerontology (TMGHIG), 35-2 Sakae-cho, Itabashi-ku, Tokyo 173- 0015, Japan. ${ }^{6}$ Seibo Hospital, 2-5-1 Nakaochiai Shinjyuku, Tokyo 161-8521, Japan}

\section{Author contributions}

T.K., M.H., and A.U. designed the study and interpreted the data; T.K., M.G., N.K. S.A., T.M., and M.I.-U. performed experiments and analysis; M.T., N.K., and T.N. performed human cell experiments; T.K., M.I.-U., M.H., and A.U. performed writing, review, and revision of the paper. All authors read and approved the final paper.

Conflict of interest

The authors declare that they have no conflict of interest.

Ethics approval and consent to participate

The experiments using human samples were approved by the Ethical Committee at Tokyo Metropolitan Geriatric Hospital and Institute of Gerontology and performed in accordance with the Declaration of Helsinki. All subjects provided their written informed consent to participate in the study. Experiments using mice were approved by the Experimental Animal Care and Use Committee of Tokyo Metropolitan Geriatric Hospital and Institute of Gerontology. 


\section{Publisher's note}

Springer Nature remains neutral with regard to jurisdictional claims in published maps and institutional affiliations.

Supplementary Information accompanies this paper at (https://doi.org/ 10.1038/s41419-020-03353-5).

Received: 12 June 2020 Revised: 14 December 2020 Accepted: 15 December 2020

Published online: 07 January 2021

\section{References}

1. Pellicoro, A., Ramachandran, P., Iredale, J. P. \& Fallowfield, J. A. Liver fibrosis and repair: immune regulation of wound healing in a solid organ. Nat. Rev. Immunol. 14, 181-194 (2014).

2. Trautwein, C., Friedman, S. L., Schuppan, D. \& Pinzani, M. Hepatic fibrosis: concept to treatment. J. Hepatol. 62, S15-S24 (2015).

3. Ebrahimi, H., Naderian, M. \& Sohrabpour, A. A. New concepts on reversibility and targeting of liver fibrosis; a review article. Middle East J. Dig. Dis. 10, 133-148 (2018).

4. Asrani, S. K., Devarbhavi, H., Eaton, J. \& Kamath, P. S. Burden of liver diseases in the world. J. Hepatol. 70, 151-171 (2019).

5. GBD_2017_Cirrhosis_Collaborators. The global, regional, and national burden of cirrhosis by cause in 195 countries and territories, 1990-2017: a systematic analysis for the Global Burden of Disease Study 2017. Lancet Gastroenterol. Hepatol. 5, 245-266 (2020).

6. D'Amico, G., Garcia-Tsao, G. \& Pagliaro, L. Natural history and prognostic indicators of survival in cirrhosis: a systematic review of 118 studies. J. Hepatol. 44, 217-231 (2006)

7. Dasarathy, S. Cause and management of muscle wasting in chronic liver disease. Curr. Opin. Gastroenterol. 32, 159-165 (2016).

8. Hanai, T. et al. Rapid skeletal muscle wasting predicts worse survival in patients with liver cirrhosis. Hepatol. Res. 46, 743-751 (2016).

9. Lucidi, C. et al. A low muscle mass increases mortality in compensated cirrhotic patients with sepsis. Liver Int. 38, 851-857 (2018).

10. Kim, G., Kang, S. H., Kim, M. Y. \& Baik, S. K. Prognostic value of sarcopenia in patients with liver cirrhosis: a systematic review and meta-analysis. PLOS ONE 12, e0186990 (2017)

11. Nishikawa, H. et al. Japan Society of Hepatology guidelines for sarcopenia in liver disease (1st edition): recommendation from the working group for creation of sarcopenia assessment criteria. Hepatol. Res. 46, 951-963 (2016).

12. Wolfe, R. R. The underappreciated role of muscle in health and disease. Am. J. Clin. Nutr. 84, 475-482 (2006).

13. Bunout, D., de la Maza, M. P., Barrera, G., Leiva, L. \& Hirsch, S. Association between sarcopenia and mortality in healthy older people. Australas. J. Ageing 30, 89-92 (2011).

14. Han, S. S. et al. Lean mass index: a better predictor of mortality than body mass index in elderly Asians. J. Am. Geriatr. Soc. 58, 312-317 (2010).

15. Wang, H., Hai, S., Liu, Y., Liu, Y. \& Dong, B. Skeletal muscle mass as a mortality predictor among nonagenarians and centenarians: a prospective cohort study. Sci. Rep. 9, 2420 (2019).

16. Landi, F. et al. Midarm muscle circumference, physical performance and mortality: results from the aging and longevity study in the Sirente geographic area (ilSIRENTE study). Clin. Nutr. 29, 441-447 (2010).

17. Cruz-Jentoft, A. J. et al. Sarcopenia: European consensus on definition and diagnosis: Report of the European Working Group on Sarcopenia in Older People. Age Ageing 39, 412-423 (2010).

18. Traub, J., Bergheim, I., Eibisberger, M. \& Stadlbauer, V. Sarcopenia and liver cirrhosis - comparison of the European Working Group on Sarcopenia Criteria 2010 and 2019. Nutrients 12, https://doi.org/10.3390/nu12020547 (2020).

19. Peterson, J. M., Bakkar, N. \& Guttridge, D. C. NF-kappaB signaling in skeletal muscle health and disease. Curr. Top. Dev. Biol. 96, 85-119 (2011).

20. Egerman, M. A. \& Glass, D. J. Signaling pathways controlling skeletal muscle mass. Crit. Rev. Biochem. Mol. Biol. 49, 59-68 (2014).

21. Garcia, P. S., Cabbabe, A., Kambadur, R., Nicholas, G. \& Csete, M. Brief-reports: elevated myostatin levels in patients with liver disease: a potential contributor to skeletal muscle wasting. Anesth. Analg. 111, 707-709 (2010).
22. Qiu, J. et al. Hyperammonemia in cirrhosis induces transcriptional regulation of myostatin by an NF-kappaB-mediated mechanism. Proc. Natl Acad. Sci. USA 110, 18162-18167 (2013).

23. Giusto, M. et al. Skeletal muscle myopenia in mice model of bile duct ligation and carbon tetrachloride-induced liver cirrhosis. Physiol. Rep. 5, https:/doi.org/ 10.14814/phy2.13153 (2017).

24. McPherron, A. C., Lawler, A. M. \& Lee, S. J. Regulation of skeletal muscle mass in mice by a new TGF-beta superfamily member. Nature 387, 83-90 (1997).

25. Cai, D. et al. IKKbeta/NF-kappaB activation causes severe muscle wasting in mice. Cell 119, 285-298 (2004).

26. Tag, C. G. et al. Bile duct ligation in mice: induction of inflammatory liver injury and fibrosis by obstructive cholestasis. J. Vis. Exp. https://doi.org/10.3791/52438 (2015).

27. Kawai, S., Takagi, Y., Kaneko, S. \& Kurosawa, T. Effect of three types of mixed anesthetic agents alternate to ketamine in mice. Exp. Anim. 60, 481-487 (2011).

28. Uezumi, A., Kasai, T. \& Tsuchida, K. Identification, isolation, and characterization of mesenchymal progenitors in mouse and human skeletal muscle. Methods Mol. Biol. 1460, 241-253 (2016).

29. Egerman, M. A. et al. GDF11 increases with age and inhibits skeletal muscle regeneration. Cell Metab. 22, 164-174 (2015).

30. Liedtke, C. et al. Experimental liver fibrosis research: update on animal models, legal issues and translational aspects. Fibrogenes. Tissue Repair 6, 19 (2013).

31. Marques, T. G. et al. Review of experimental models for inducing hepatic cirrhosis by bile duct ligation and carbon tetrachloride injection. Acta Cir. Bras. 27, 589-594 (2012).

32. Turpin, S. M., Lancaster, G. I., Darby, I., Febbraio, M. A. \& Watt, M. J. Apoptosis in skeletal muscle myotubes is induced by ceramides and is positively related to insulin resistance. Am. J. Physiol. Endocrinol. Metab. 291, E1341-E1350 (2006).

33. Bryner, R. W., Woodworth-Hobbs, M. E., Williamson, D. L. \& Alway, S. E. Docosahexaenoic acid protects muscle cells from palmitate-induced atrophy. ISRN Obes. 2012, 647348 (2012).

34. Turpin, S. M. et al. Examination of 'lipotoxicity' in skeletal muscle of high-fat fed and ob/ob mice. J. Physiol. 587, 1593-1605 (2009).

35. Enochsson, L., Isaksson, B., Strömmer, L., Erlanson-Albertsson, C. \& Permert, J. Bile duct obstruction is associated with early postoperative upregulation of liver uncoupling protein-2 and reduced circulating glucose concentration in the rat. Nutrition 26, 405-410 (2010).

36. Machado, L. et al. In situ fixation redefines quiescence and early activation of skeletal muscle stem cells. Cell Rep. 21, 1982-1993 (2017).

37. Hoffmann, R., Grewe, M., Estler, H. C., Schulze-Specking, A. \& Decker, K. Regulation of tumor necrosis factor-alpha-mRNA synthesis and distribution of tumor necrosis factor-alpha-mRNA synthesizing cells in rat liver during experimental endotoxemia. J. Hepatol. 20, 122-128 (1994).

38. Fox, E. S., Kim, J. C. \& Tracy, T. F. NF-kappaB activation and modulation in hepatic macrophages during cholestatic injury. J. Surg. Res. 72, 129-134 (1997).

39. Canbay, A. et al. Kupffer cell engulfment of apoptotic bodies stimulates death ligand and cytokine expression. Hepatology 38, 1188-1198 (2003).

40. Mederacke, I. et al. Fate tracing reveals hepatic stellate cells as dominant contributors to liver fibrosis independent of its aetiology. Nat. Commun. 4, 2823 (2013).

41. Bataller, R. et al. Systemic infusion of angiotensin II exacerbates liver fibrosis in bile duct-ligated rats. Hepatology 41, 1046-1055 (2005).

42. Thirunavukkarasu, C., Watkins, S. C. \& Gandhi, C. R. Mechanisms of endotoxininduced $\mathrm{NO}, \mathrm{IL}-6$, and TNF-alpha production in activated rat hepatic stellate cells: role of p38 MAPK. Hepatology 44, 389-398 (2006).

43. Friedman, S. L. Hepatic stellate cells: protean, multifunctional, and enigmatic cells of the liver. Physiol. Rev. 88, 125-172 (2008).

44. Meng, F. et al. Interleukin-17 signaling in inflammatory, Kupffer cells, and hepatic stellate cells exacerbates liver fibrosis in mice. Gastroenterology 143 765-776.e763 (2012).

45. Chen, W. et al. Activation of autophagy is required for Oroxylin A to alleviate carbon tetrachloride-induced liver fibrosis and hepatic stellate cell activation. Int. Immunopharmacol. 56, 148-155 (2018).

46. Hamilton, T. G., Klinghoffer, R. A., Corrin, P. D. \& Soriano, P. Evolutionary divergence of platelet-derived growth factor alpha receptor signaling mechanisms. Mol. Cell Biol. 23, 4013-4025 (2003).

47. Tabula Muris Consortium, A. single-cell transcriptomic atlas characterizes ageing tissues in the mouse. Nature 583, 590-595 (2020). 
48. Li, Y. P. et al. TNF-alpha acts via p38 MAPK to stimulate expression of the ubiquitin ligase atrogin1/MAFbx in skeletal muscle. FASEB J. 19, 362-370 (2005).

49. Gordon, J. N. et al. Thalidomide in the treatment of cancer cachexia: a randomised placebo controlled trial. Gut 54, 540-545 (2005).

50. Furrer, R. \& Handschin, C. Muscle wasting diseases: novel targets and treatments. Annu. Rev. Pharm. Toxicol. 59, 315-339 (2019).

51. Sudo, K., Yamada, Y., Moriwaki, H., Saito, K. \& Seishima, M. Lack of tumor necrosis factor receptor type 1 inhibits liver fibrosis induced by carbon tetrachloride in mice. Cytokine 29, 236-244 (2005).

52. Sundaram, B. et al. iRhom 2 inhibits bile duct obstruction-induced liver fibrosis. Sci. Signal. 12, https://doi.org/10.1126/scisignal.aax1194 (2019).

53. $\mathrm{Wu}, \mathrm{H}$. et al. Single-cell transcriptomics of a human kidney allograft biopsy specimen defines a diverse inflammatory response. J. Am. Soc. Nephrol. 29, 2069-2080 (2018)

54. MacParland, S. A. et al. Single cell RNA sequencing of human liver reveals distinct intrahepatic macrophage populations. Nat. Commun. 9, 4383 (2018).
55. Nguyen, Q. H., Pervolarakis, N., Nee, K. \& Kessenbrock, K. Experimental considerations for single-cell RNA sequencing approaches. Front. Cell Dev. Biol. 6 , 108 (2018).

56. Wagner, K. R. et al. A phase I/lltrial of MYO-029 in adult subjects with muscular dystrophy. Ann. Neurol. 63, 561-571 (2008).

57. Latres, E. et al. Activin A more prominently regulates muscle mass in primates than does GDF8. Nat. Commun. 8, 15153 (2017).

58. Zimmers, T. A. et al. Exogenous GDF11 induces cardiac and skeletal muscle dysfunction and wasting. Basic Res. Cardiol. 112, 48 (2017).

59. Lee, S. J. Quadrupling muscle mass in mice by targeting TGF-beta signaling pathways. PLoS ONE 2, e789 (2007).

60. Dasarathy, S. \& Merli, M. Sarcopenia from mechanism to diagnosis and treatment in liver disease. J. Hepatol. 65, 1232-1244 (2016).

61. Davuluri, G. et al. Metabolic adaptation of skeletal muscle to hyperammonemia drives the beneficial effects of H-leucine in cirrhosis. J. Hepatol. 65, 929-937 (2016). 